\title{
Crystal Structure Of The UDP-Glucose Pyrophosphorylase From Yersinia Pestis, a Drug Target for New Anti-Plague Agents
}

George T. Lountos ${ }^{1}$, Morgan E. Gibbs ${ }^{2}$, Rajesh Gumpena ${ }^{2}$, and David S. Waugh ${ }^{2}$ ${ }^{1}$ Basic Science Program, Frederick National Laboratory for Cancer Research, Frederick, MD, and

${ }^{2}$ Macromolecular Crystallography Laboratory, National Cancer Institute, Frederick, MD

Yersinia pestis, the causative agent of bubonic plague, is labeled as a high-threat, category $\mathrm{A}$ bioterrorism agent by the Center for Disease Control. Today, a limited number of antibiotics are used for treatment but drug-resistant isolates of $Y$. pestis have arisen in a few areas of the world. Despite the very low level of clinically reported cases of plague, the main concern around $Y$. pestis is its possible misuse as a bioterrorism agent. Therefore, the discovery of new drugs that can be rapidly accessed in such an emergency warrants continued research. The UDP-glucose pyrophosphorylase (UGP) from $Y$. pestis is an enzyme that has been linked to the survival of $Y$. pestis in mouse macrophages. Here, we report the crystal structure of the UDPglucose pyrophosphorylase from $Y$. pestis at $2.17 \AA$ resolution which reveals high structural homology with other reported bacterial UGPs. Bacterial UGPS share high sequence conservation of the active site but exhibit significant sequence divergence with their eukaryotic UGP counterparts. Therefore, the development of broad-spectrum inhibitors that target bacterial UGPs might be feasible while, at the same time, avoiding off-target effects in the eukaryotic host. The crystal structure reported here provides the first step to guide the rational design of new UGP inhibitors.

George Lountos lountosg@mail.nih.gov

Morgan Gibbs gibbsm@live.unc.edu

Rajesh Gumpena rajesh2810@yahoo.co.in

David Waugh waughd@mail.nih.gov 